\title{
Specific DNA mini-barcoding for identification of Gekko gecko and its products
}

\author{
Yanyan Su ${ }^{1,2+}$, Dandan Ding ${ }^{1+}$, Mengjie Yao ${ }^{3}$, Lan Wu ${ }^{1}$, Gangqiang Dong ${ }^{2}$, Dong Zhang ${ }^{1}$, Shilin Chen ${ }^{1 *}$ \\ and Li Xiang ${ }^{1 *}$
}

\begin{abstract}
Background: The dry body of the Tokay Gecko (Gekko gecko) is the source of a valuable traditional Chinese medicine, it is therefore listed as a Class II protected animal species in China. Due to increasing market demand and a declining supply of the species, a considerable number of adulterants have emerged in the market. Thus, it is necessary to establish an accurate and rapid method of identification for distinguishing G. gecko from its adulterants and for separating it from highly processed products.

Methods: A total of $274 \mathrm{CO}$ / sequences were analyzed by using MEGA 5.0 software. Several specific primers were designed to amplify mini-barcode regions and identify G. gecko from its counterfeits and products.

Results: $274 \mathrm{CO}$ sequences of G. gecko and 15 adulterants species were analyzed. G. gecko could be distinguished from its adulterants through BLAST analysis, intra- and inter-specific distance analyses, and an NJ tree based on COI sequences. Two pairs of specific primers designed for this study, COISF2/COISR2 and COISF3/COISR3, amplified 200and 133-bp fragments of the $\mathrm{COI}$ region, respectively, both of which were suitable for the identification of G. gecko and its adulterants. Furthermore, COISF3/COISR3 detected G. gecko in 15 batches of products.

Conclusion: Therefore, the specific DNA mini-barcoding method developed here may be a powerful tool for the identification of G. gecko and counterfeits, and may also be used to distinguish G. gecko from its highly processed by-products.
\end{abstract}

Keywords: Gekko gecko, specific primers, DNA mini-barcoding, Identification

\section{Background}

As one of the rarest Chinese medicinal materials, the dry body of Tokay Gecko (G. gecko Linnaeus), has been used for two thousand years and is known for its remarkably therapeutic effect on kidney deficiency [1,2]. Previous studies have found that G. gecko also has notable effects in relieving asthma, strengthening the immune system,

\footnotetext{
*Correspondence: slchen@icmm.ac.cn; lxiang@icmm.ac.cn

${ }^{\dagger}$ Yanyan Su and Dandan Ding contributed equally to this work

${ }^{1}$ Institute of Chinese Materia Medica, China Academy of Chinese Medical Sciences, Beijing 100700, China

Full list of author information is available at the end of the article
}

and treating tumors [3-5]. Today it is widely used in many functional foods and Chinese patent medicines, such as tinctures, Renshen Gejie Powder, Gejie Dingchuan Capsule, Gejie Dingchuan Pill, and Shenge Pingchuan Capsule [6, 7]. For decades, the medicinal demand for G. gecko has skyrocketed in China. However, due to the destruction of its natural habitats and indiscriminate hunting, the wild G. gecko has been classified as a Class II protected animal species in China since 1989 [8]. As a result, the demand for the animal is considerably higher than its supply. Currently, the domestic market relies on imported Tokay Gecko from Southeast Asia and nativecultured G. gecko [9]. Increasing market demand and 
the declining supply of the native G. gecko increases its value; consequently, a considerable number of adulterants have emerged in the market, including Gekko swinhonis, Hemidactylus frenatus, Laudakia himalayana, Eumeces chinensis, Batrachuperus pinchonii, and other common adulterants [10-12]. Besides their very similar appearances, their ambiguous common names have also exacerbated the difficulty in distinguishing G. gecko from counterfeits. For example, G. swinhonis is commonly known as little Gecko, and L. himalayana is known as the Tibet Gecko [13]. The indistinguishable adulterants infiltrate the markets and severely damage public safety. Thus, an efficient identification method needs to be urgently developed.

At present, the most frequently used method of identifying G. gecko involves observing its morphological characteristics and conducting microscopic analyses [14-16]; it is not practical for researchers without knowledge of taxonomy to differentiate between incomplete samples and powders based on morphological characteristics [17-19]. No reliable method of identification has been reported because no specific chemical component functioning as a chemical marker has been found. It is unclear what active component of G. gecko is used in related functional foods and Chinese patent medicines [20,21]. Although DNA barcoding technology based on COI region has the ability to distinguish G. gecko from related species and to identify other animal species successfully $[11,22,23]$, this technology cannot be applied to samples with degraded DNA because it is impossible to amplify the COI region (>600 bp) of highly processed materials [24-27].

Therefore, a new method using DNA mini-barcoding based on the COI region combined with a specific PCR technique was invented and used in this study to distinguish G. gecko from its adulterants and related products.

\section{Materials and methods Materials}

All experiments were performed in accordance with the guidelines for the use and care of animals of the Center for Laboratory Animal Care, China Academy of Chinese Medical Sciences. All experimental protocols were approved by the Research Ethics Committee of Institute of Chinese Materia Medica, China Academy of Chinese Medical Sciences, Beijing, China. A total of 269 samples, including 47 original animals (dry samples from local stores), 19 specimens (dry and dissected samples from Kunming Institute of Zoology, Chinese Academy of Sciences), and 203 medicinal materials belonging to 16 species of G. gecko and its adulterants were collected from localities, such as Anguo herb market and Bozhou herb market (see Fig. 1 and Additional file 1: Table S1). A total of 274 COI sequences were analyzed, including 269 sequences obtained from this study and five sequences downloaded from GenBank. All the samples of this study were deposited as Voucher at the Institute of Chinese Materia Medica, China Academy of Chinese Medical Sciences, and the obtained 269 COI sequences have been submitted to GenBank.

In addition, 15 batches of five kinds of related products were purchased from online stores and drugstores.

\section{DNA extraction, amplification, and sequencing}

In $75 \%$ ethanol $10-30 \mathrm{mg}$ of underlying muscles of specimens were rinsed then pulverized using a DNA Extraction Grinder (Sceintz Biotech Co., China). Genomic DNA was extracted using an Animal Genomic DNA Kit (Tiangen Biotech Co., Ltd, Beijing, China). COI sequences were amplified with universal primers LCO1490/HCO2198 according to Standard DNA Barcodes of Chinese Materia Medica in Chinese Pharmacopoeia $[11,28,29]$. The PCR products were examined using $1.0 \%$ agarose gel electrophoresis. The purified PCR products were bi-directionally sequenced using an $\mathrm{ABI}$ 3730 XL sequencer (Applied Biosystems Inc.).

\section{Sequence analysis and primers design}

The attained trace files were assembled and trimmed using CodonCode Aligner V 3.0 (CondonCode Co., USA). Then the 269 COI sequences were verified in the NCBI database and DNA Barcoding System for Identifying Herbal Medicine (http://www.tcmbarcode.cn/ en/). A total of 274 COI sequences were analyzed using MEGA 5.0 software, including calculating intra- and inter-specific distances and constructing a neighbor-joining (NJ) tree based on the Kimura 2-Parameter model. Sequentially, two pairs of specific primers of G. gecko were designed based on COI regions of G. gecko (658 bp) using Primer 5.0 software: COISF2: 5'-GCCTCCGCG AGTGTG-3', COISR2: 5'-CGTGTATTGGGTTATGCT TGG-3'; and COISF3: 5'-AAAATGAAAACCCCAAG3', COISR3: 5'-TACGAACGGTCAACAA-3'. A reaction mixture comprising of $12.5 \mu \mathrm{L} 2 \times$ Taq PCR Master Mix (Aidlab Biotechnologies Co., China), $1 \mu \mathrm{L}$ of each primer $(2.5 \mu \mathrm{M}), 1 \mu \mathrm{L}$ genomic DNA, and $9.5 \mu \mathrm{L} \mathrm{ddH}_{2} \mathrm{O}$ was prepared. The PCR amplification conditions of COISF2/ COISR2 were initially set up as $94{ }^{\circ} \mathrm{C}$ for $5 \mathrm{~min}$, followed by 40 cycles of $94{ }^{\circ} \mathrm{C}$ for $30 \mathrm{~s}, 61^{\circ} \mathrm{C}$ for $30 \mathrm{~s}$, and $72{ }^{\circ} \mathrm{C}$ for $30 \mathrm{~s}$, and a final extension at $72{ }^{\circ} \mathrm{C}$ for $10 \mathrm{~min}$. The amplification of COISF3/COISR3 started with $94^{\circ} \mathrm{C}$ for $5 \mathrm{~min}$, followed by 40 cycles of $94{ }^{\circ} \mathrm{C}$ for $30 \mathrm{~s}, 50^{\circ} \mathrm{C}$ for $30 \mathrm{~s}$, and $72{ }^{\circ} \mathrm{C}$ for $30 \mathrm{~s}$, and a final extension temperature at $72{ }^{\circ} \mathrm{C}$ for $5 \mathrm{~min}$.

The genomic DNA extracted from G. gecko was diluted to a series of concentrations $(100 \mathrm{ng} / \mu \mathrm{L}, 10 \mathrm{ng} / \mu \mathrm{L}, 1 \mathrm{ng} /$ 

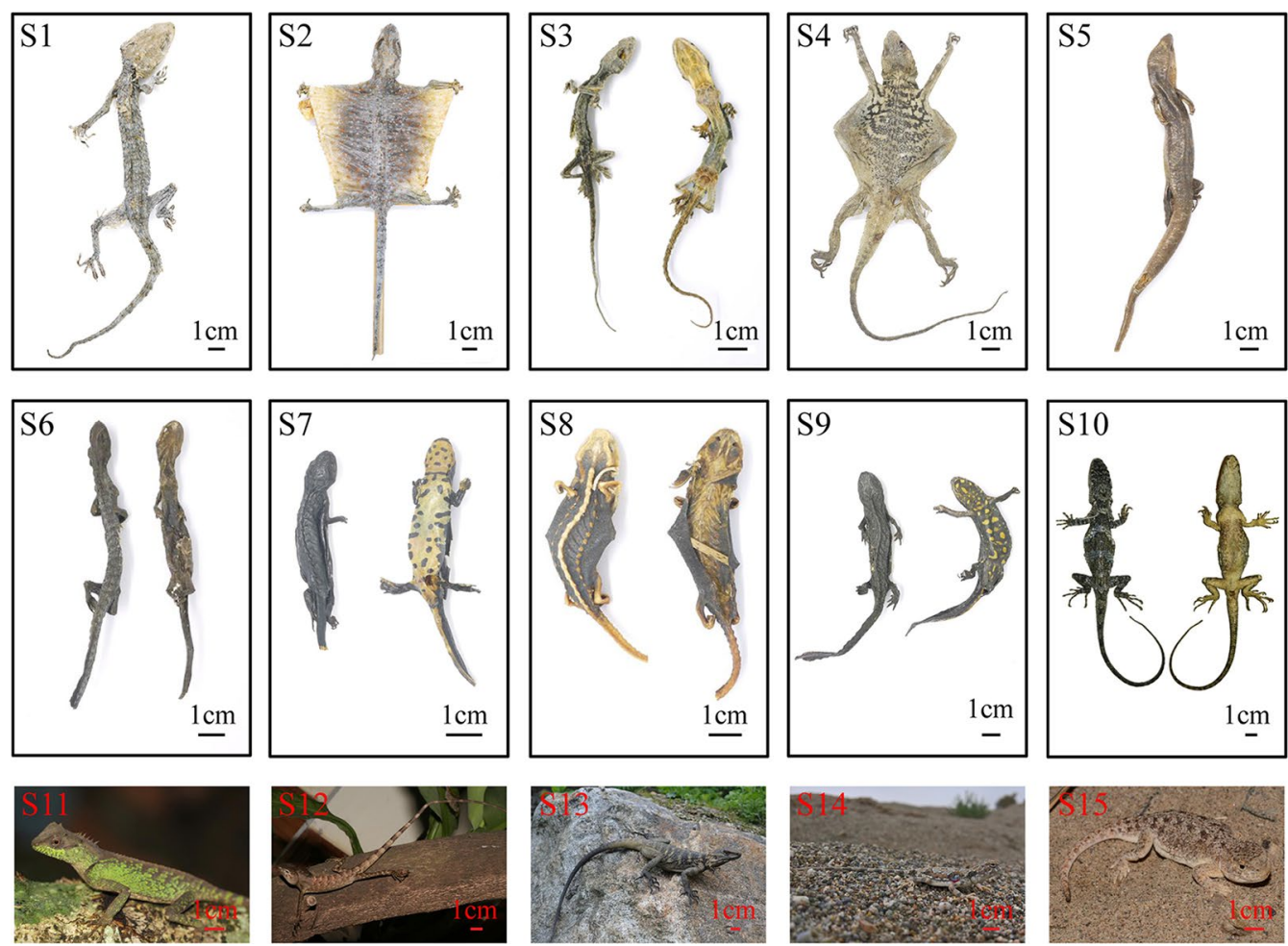

Fig. 1 Photos of samples in this study. S1, S2: G. gecko, S3: G. swinhonis, S4: L. himalayana, S5: E. chinensis, S6: B. pinchonii, S7: C. orientalis, S8: T. shanjing, S9: P. chinensis, S10: C. kakhienensis, S11: A. lepidogaster, S12: C. versicolor, S13: L. sacra, S14: P. axillaris and S15: P. theobaldi

$\mu \mathrm{L}, 100 \mathrm{pg} / \mu \mathrm{L}, 10 \mathrm{pg} / \mu \mathrm{L}$, and $1 \mathrm{pg} / \mu \mathrm{L})$ and then amplified with the specific primers to determine the minimum amplifiable concentration.

\section{Distinguishing G. gecko from related products}

Fifteen batches of functional foods and Chinese patent medicines, including three batches of functional foods (Renshen Gejie Powder), twelve batches of Chinese patent medicines (three batches of Gejie Dingchuan Pill, Gejie Dingchuan Capsule, Shenge Pingchuan Capsule, and Gejie Dangshen Syrup each), allegedly containing $G$. gecko were collected from online stores and drugstores. DNA was extracted from all samples in triplicates. Using nuclear separation liquid (100 mM Tris- $\mathrm{HCl}(\mathrm{pH} 8.0)$, $20 \mathrm{mM}$ EDTA (pH 8.0), $700 \mathrm{mM} \mathrm{NaCl}, 2 \%$ PVP-40, and $0.4 \% \beta$-mercaptoethanol), $0.5-1.0 \mathrm{~g}$ of powdered samples, 5-6 g of pills, and $10 \mathrm{~g}$ of syrup were rinsed 1-5 times until the supernatant liquid became lightly colored or colorless [30]. Genomic DNA of these functional foods and Chinese patent medicines was extracted using an Animal Genomic DNA Kit (Tiangen Biotech Co., Ltd, Beijing, China) and amplified with universal primers of $\mathrm{COI}$ region and specific primers to examine the existence of G. gecko. A total of 15 DNA samples were detected with PCR reaction, three samples of each product.

\section{Results}

\section{Identification of G. gecko from adulterants through DNA barcoding}

In this study, $274 \mathrm{COI}$ sequences belonging to 16 species were analyzed after being verified in the NCBI database and DNA Barcoding System for Identifying Herbal Medicine (see Additional file 1: Table S1). The results are shown in Additional file 1: Table S2. The COI sequences for most species were $658 \mathrm{bp}$ long, and $P$. axillarisone was the species with the shortest COI sequence (611 bp). The guanine-cytosine $(\mathrm{GC})$ content for all species was lower than $50 \%$ except for H. frenatus (50.6\%); G. gecko had a GC content of $48.2 \%$ and B. pinchonii had the lowest GC content (39.5\%). Furthermore, the maximum intra-specific K2P distances between G. gecko and 15 adulterants were less than their minimum inter-specific distances, which indicated that every species in this study could be distinguished from other species (see Additional file 1: Table S2). In addition, an NJ tree was constructed using 69 haplotypes of $274 \mathrm{COI}$ sequences which belong to 16 species (see Additional file 1: Figure S1). The haplotypes of G. gecko clustered into a branch with a bootstrap value of 99 , and the haplotypes of counterfeits also formed branches with high bootstrap values. This result correlated with that of the intra- and inter-specific distances 
analyses suggesting that DNA barcoding could effectively distinguish G. gecko from other related species.

\section{Design and amplification of specific primers}

Two specific primers COISF2/COISR2 (COISF2: 5'-GCC TCCGCGAGTGTG-3'， COISR2: 5'-CGTGTATTG GGTTATGCTTGG-3') and COISF3/COISR3 (COISF3: 5'-AAAATGAAAACCCCAAG-3', COISR3: 5'-TACGAA CGGTCAACAA-3') amplified 200- and 133-bp fragments of the $C O I$ region respectively, and were designed for the mini-barcoding of G. gecko and to identify related products (Additional file 1: Figures S2 and S3). The universal $C O I$ primers and two specific primers were used in this study to amplify genomic DNA of G. gecko and its 15 adulterants. Using universal primers of COI, all samples were successfully amplified and showed markers approximately 750 bp long (Fig. 2a). We found that the template DNA of specimens met the requirement of amplification. Furthermore, when specific primers were used, only
G. gecko showed expected markers at 200 and $133 \mathrm{bp}$; however, no other target PCR products could be seen (Fig. 2b, c). The results confirmed that the two primer pairs COISF2/COISR2 and COISF3/COISR3 developed in this study could effectively and distinctly separate $G$. gecko from its adulterants.

\section{Sensitivity of specific primers}

To examine the sensitivity of the two specific primers, template DNA was diluted to a series of concentrations varying from $100 \mathrm{ng} / \mu \mathrm{L}$ to $1 \mathrm{pg} / \mu \mathrm{L}$. Then, these samples were amplified with three replicates each using universal primers, LCO1490/HCO2198 and the two specific primers, COISF2/COISR2 and COISF3/COISR3 (Fig. 3). The minimum effective concentrations of DNA that could be amplified by these primers were $10 \mathrm{pg} / \mu \mathrm{L}$. More specifically, when the template DNA concentration was only $1 \mathrm{pg} / \mu \mathrm{L}$, the band of PCR products with primers COISF3/COISR3 were faintly visible (Fig. 3c). Specific

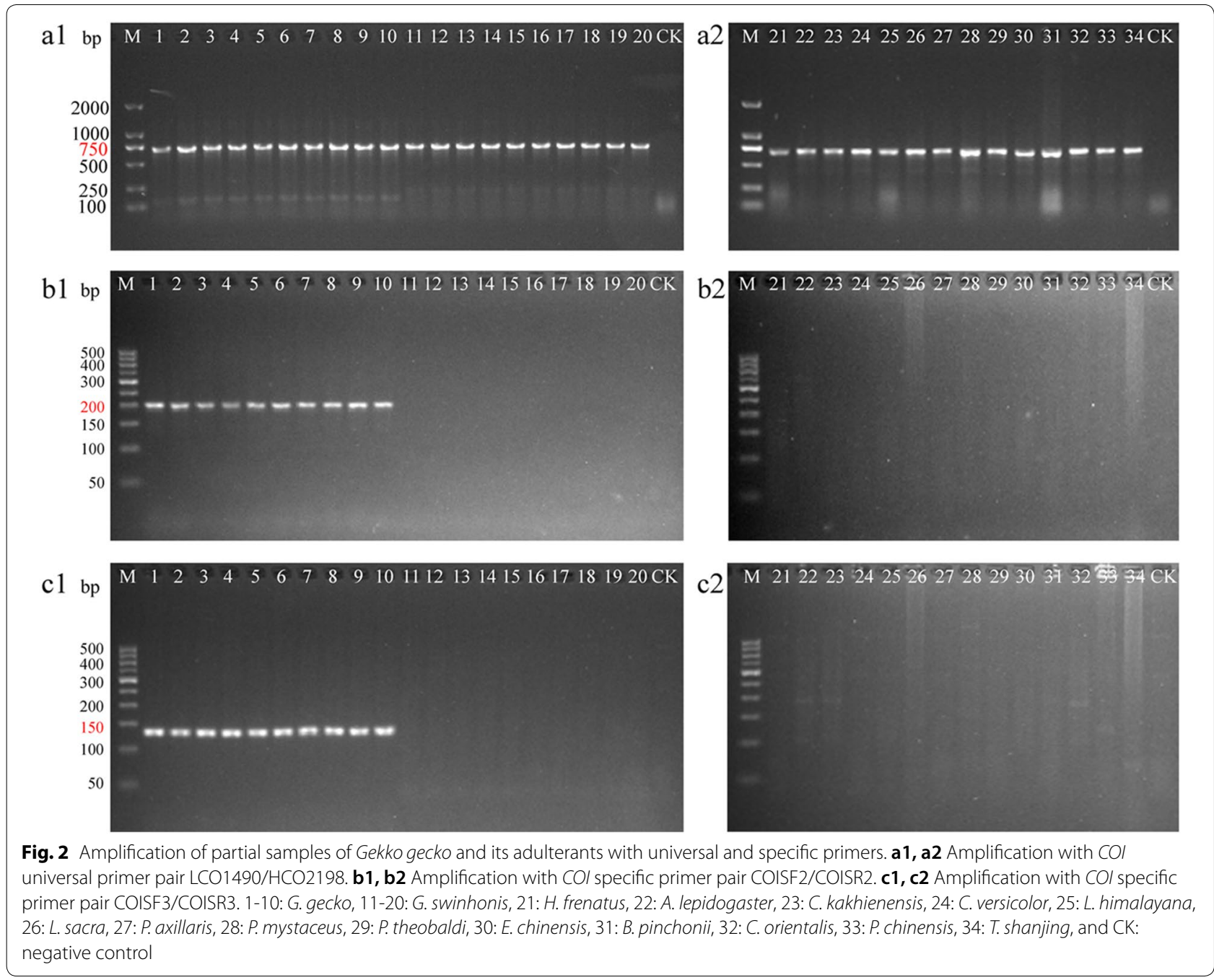



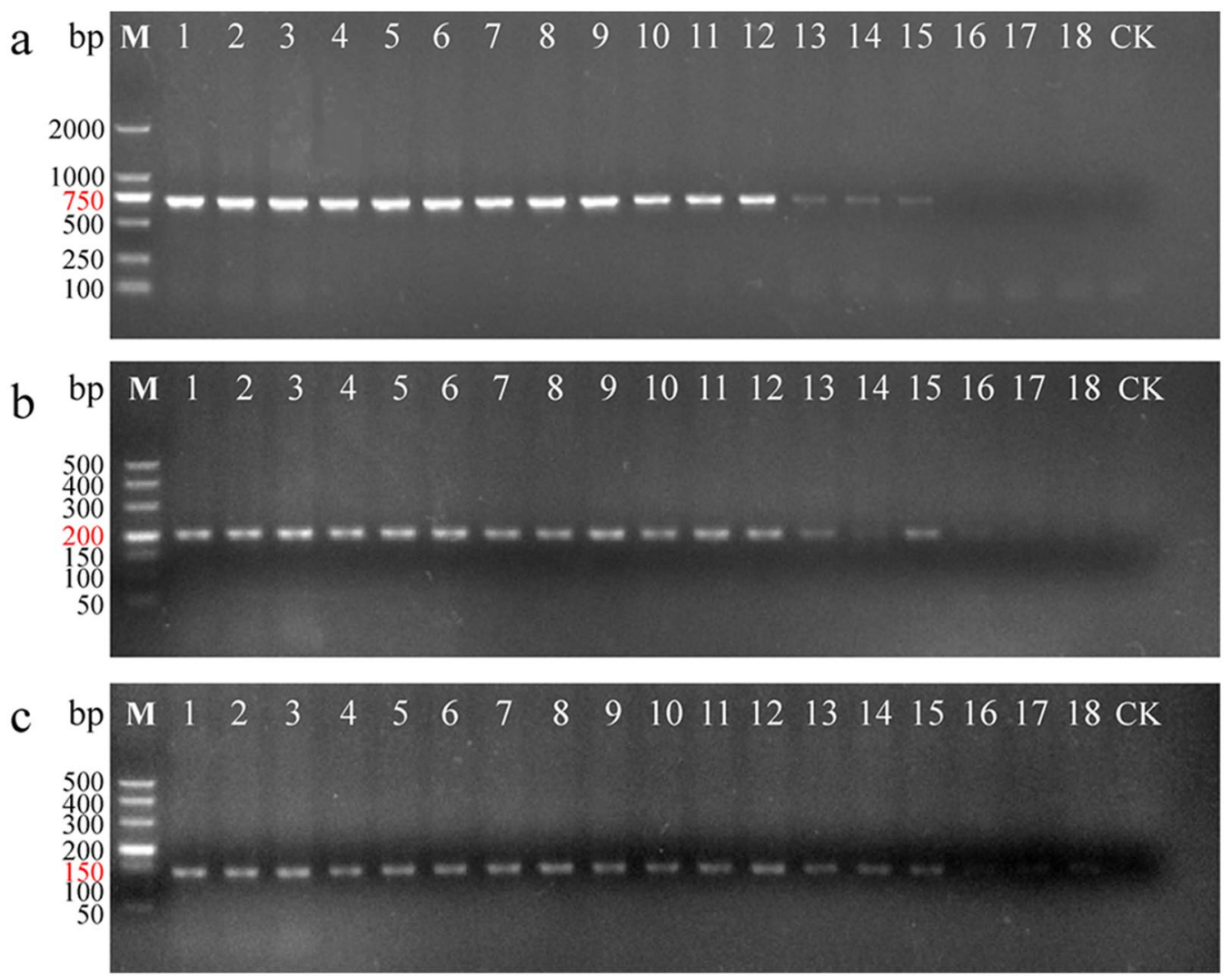

Fig. 3 The sensitivity of amplification with universal and specific primers for detection of Gekko gecko. a Amplification with CO/ universal primer pair LCO1490/HCO2198. b Amplification with COI specific primer pair COISF2/COISR2. c Amplification with COI specific primer pair COISF3/COISR3. 1-3: $100 \mathrm{ng} / \mu \mathrm{L}, 4-6: 10 \mathrm{ng} / \mu \mathrm{L}, 7-9: 1 \mathrm{ng} / \mu \mathrm{L}, 10-12: 100 \mathrm{pg} / \mu \mathrm{L}, 13-15: 10 \mathrm{pg} / \mu \mathrm{L}, 16-18: 1 \mathrm{pg} / \mu \mathrm{L}$, and CK: negative control

primers COISF3/COISR3 were more sensitive to G. gecko than the universal primer and the required minimal template concentration was $1-10 \mathrm{pg} / \mu \mathrm{L}$.

\section{Distinguishing G. gecko from its related products}

To verify the utility and practicability of the specific primers, DNA extracted from samples obtained from 15 batches of functional foods were amplified with the universal and specific primers (Table 1). Only G. gecko from Renshen Gejie Powder could be successfully amplified with universal primers LCO1490/HCO2198 (Additional file 1: Figures S4a). Using the specific primers COISF2/COISR2, G. gecko was successfully amplified from Renshen Gejie Powder and Shenge Pingchuan Capsule (Additional file 1: Figures S4b). For specific primers COISF3/COISR3, the amplification results of five kinds of related products in this study were positive (Additional file 1: Figures S4c). All the PCR products were sequenced to ensure the reliability of amplification, and obtained sequences were verified in the NCBI database and DNA Barcoding System for Identifying Herbal Medicine. The results revealed that all 15 batches of related products in this study contained G. gecko. Additionally, it was confirmed that COISF3/COISR3 could be used to distinguish G. gecko from its related products.

\section{Discussion}

Due to over-exploitation of G. gecko as a traditional Chinese medicine, it has been listed as a Class II protected animal species. As a result, the demand has always greatly exceeded market supply and various adulterants have emerged in the market, which compromise clinical effects and public safety.

In this study, G. gecko and its 15 adulterants were distinguished from each other through DNA barcoding based on COI sequences. The results were consistent with a previously reported study that identified G. gecko from adulterants using COI regions [11, 22]. DNA minibarcoding and specific-PCR identification are becoming more popular methods for effective species identification $[24,25,31]$, especially for specimens with degraded DNA, such as archival specimens, processed foods, functional foods, and Chinese patent medicines [26, 27, 32-35]. For a more sensitive identification of G. gecko in 
Table 1 Detection results of Gekko gecko from related products

\begin{tabular}{|c|c|c|c|c|c|c|}
\hline \multirow[t]{2}{*}{ Sample no. } & \multirow[t]{2}{*}{ Product name } & \multirow{2}{*}{$\begin{array}{l}\text { Ingredients } \\
\text { number }\end{array}$} & \multirow[t]{2}{*}{ Repetitions } & \multicolumn{3}{|c|}{ Amplification results } \\
\hline & & & & $\begin{array}{l}\text { LCO1490/ } \\
\text { HCO2198 }\end{array}$ & $\begin{array}{l}\text { COISF2/ } \\
\text { COISR2 }\end{array}$ & $\begin{array}{l}\text { COISF3/ } \\
\text { COISR3 }\end{array}$ \\
\hline RGS1 & Renshen Gejie Powder & 8 & 3 & Y & Y & Y \\
\hline RGS2 & Renshen Gejie Powder & 8 & 3 & Y & Y & Y \\
\hline RGS3 & Renshen Gejie Powder & 8 & 3 & Y & Y & Y \\
\hline SGPC1 & Shenge Pingchuan Capsule & 4 & 3 & N & Y & Y \\
\hline SGPC2 & Shenge Pingchuan Capsule & 4 & 3 & N & Y & Y \\
\hline SGPC3 & Shenge Pingchuan Capsule & 4 & 3 & N & Y & Y \\
\hline GDG1 & Gejie Dangshen Syrup & 2 & 3 & N & N & Y \\
\hline GDG2 & Gejie Dangshen Syrup & 2 & 3 & N & N & Y \\
\hline GDG3 & Gejie Dangshen Syrup & 2 & 3 & N & N & Y \\
\hline DCW1 & Gejie Dingchuan Pill & 14 & 3 & N & N & Y \\
\hline DCW2 & Gejie Dingchuan Pill & 14 & 3 & N & $\mathrm{N}$ & Y \\
\hline DCW3 & Gejie Dingchuan Pill & 14 & 3 & N & N & Y \\
\hline DCJN1 & Gejie Dingchuan Capsule & 14 & 3 & N & $\mathrm{N}$ & Y \\
\hline DCJN2 & Gejie Dingchuan Capsule & 14 & 3 & N & N & Y \\
\hline DCJN3 & Gejie Dingchuan Capsule & 14 & 3 & $\mathrm{~N}$ & $\mathrm{~N}$ & Y \\
\hline
\end{tabular}

Y means target fragments were successfully amplified. $\mathrm{N}$ implies no fragments were amplified

2001, it was distinguished from 14 adulterants through allele-specific diagnostic PCR which amplified 260-bp fragments of the $12 \mathrm{~S}$ rRNA gene [36]. To distinguish $G$. gecko from 11 adulterants, $\mathrm{Gu}$ proposed four specific primer pairs according to the COI, Cytb, 16S, and $12 \mathrm{~S}$ rRNA gene sequences of G. gecko and amplified 210-, 200-, 345-, and 110-bp fragments, respectively [37]. Jiang applied a specific PCR method to amplify approximately 400-bp fragment of the COI region to identify G. gecko from seven common adulterants [38]. Although significant efforts have been made by researchers, degraded DNA extracted from G. gecko-relevant functional foods and Chinese patent medicines have yet to be tested.

In this study, specific primers COISF2/COISR2 and COISF3/COISR3 were designed to amplify mini-barcode. The primers could amplify 200- and 133-bp COI fragments, respectively. The specific primers COISF3/ COISR3 can be used not only for the identification of original animal of G. gecko from its adulterants, but also for the identification of functional foods and Chinese patent medicines. Similar to other DNA mini-barcoding studies, in which 100-150 bp fragments were amplified with specific primers to distinguish animal species from Alheira sausages and processed fins $[39,40]$, our results showed that only 133-bp fragments were successfully amplified with template DNA extracted from 15 batches of products. This suggested that the DNA obtained from highly processed products were highly degraded. Hence, specific-PCR combined with DNA mini-barcoding may be a powerful tool for verifying labeling compliance and evaluating the possible existence of fraudulent practices [39, 41-43]. In addition, it is useful for identification of archival specimens and expands the application of DNA barcoding.

It is important to note that more studies concentrated on the distinctions between black-spotted and redspotted Tokay Geckos. Black-spotted Tokay Geckos are the authentic medical material while red-spotted Tokay Geckos are a substitute $[1,44]$. Numerous studies show significant differences between the black-spotted and red-spotted Tokay Geckos based on vocalization analyses, 12S rRNA gene sequences, complete mitochondrial genome and mitochondrial cytochrome $b$ gene sequences, nuclear DNA information, karyotypes, and microsatellite loci analyses [45-50]. Furthermore, Qin, Zhang, and Kongbuntad found that G. gecko were genetically diverse in relation to their geographical distribution [51-53]. Furthermore, two types of G. gecko sp. may have subspecies, despite the fact that the red-spotted Tokay Gecko has been classified as G. gecko in Chinese Pharmacopoeia since 2010 [54]. At present, due to limited specimens and comprehensive data, there is still not enough evidence to support the viewpoint that black-spotted Tokay Gecko and red-spotted Tokay Gecko are different species. Specific primers COISF3/COISR3 from this study may be applied to amplify old museum specimens and acquire more information for the revision of the $G$. gecko phylogeny.

The specific primers are sensitive identifiers of samples with and without the Tokay Gecko but fail to recognize 
admixtures. Pentaplex PCR technology and DNA barcoding techniques, combined with high resolution melting (Bar-HRM method), can detect adulterants in admixtures based on mini-barcoding with universal primers efficiently [55-59]. However, the variable sites of the COI sequence of the 16 species in this study were so many that universal primers of mini-barcoding could be developed successfully. Further research is required to propose a suitable region for Bar-HRM identification based on mini-barcoding with universal primers.

\section{Conclusion}

In this study, G. gecko and its 15 adulterants were distinguished from each other through DNA barcoding based on $C O I$ sequences. Two pairs of specific primers COISF2/COISR2 and COISF3/COISR3 of G. gecko were designed to amplify 200-bp and 133-bp fragments of COI region, both of which were suitable for the identification of G. gecko and its adulterants. Importantly, specific primers COISF3/COISR3 could distinguish G. gecko from its products. Consequently, the specific DNA mini-barcoding method established from this study is a powerful tool for the identification of G. gecko and its adulterants. Therefore, this method could complement the traditional identification methods and lead to the selective identification of G. gecko from highly processed products. This study also provides insight into the identification of Chinese patent medicines.

\section{Supplementary information}

Supplementary information accompanies this paper at https://doi. org/10.1186/s13020-020-00382-2.

\begin{abstract}
Additional file 1. Information of Gekko gecko and its adulterants, $\mathrm{CO}$ sequences analysis, figures of amplification of Gekko gecko from related products with $\mathrm{CO} /$ universal primers and specific primers. Table S1. The information of Gekko gecko and its adulterants in this study. Table S2. COI sequences characteristics and K2P distances of Gekko gecko and its adulterants. Figure $\mathbf{S 1}$. The Neighbor-joining (NJ) tree based on haplotypes of Gekko gecko and its adulterants' COI sequences. The bootstrap values (1000 replicates) were showed ( $\geq 50 \%)$ for each branch. The number of samples producing each haplotype was noted in "( )". Figure S2. Alignment of specific primers COISF2/COISR2 binding regions of G. gecko and adulterants. The number of samples producing each haplotype was noted in " ( )". Figure S3. Alignment of specific primers COISF3/COISR3 binding regions of $G$. gecko and adulterants. The number of samples producing each haplotype was noted in "( )". Figure S4. Amplification of Gekko gecko from related products. a Amplification with COI universal primer pair LCO1490/ HCO2198. b Amplification with CO/ specific primer pair COISF2/COISR2. c Amplification with COI specific primer pair COISF3/COISR3. 1-3: Renshen Gejie Powder, 4-6: Shenge Pingchuan Capsule, 7-9: Gejie Dangshen Syrup, 10-12: Gejie Dingchuan Pill, 13-15: Gejie Dingchuan Capsule, CK: negative control.
\end{abstract}

\section{Acknowledgements}

We thank Ke Jiang, the researcher of Kunming Institute of Zoology, Chinese Academy of Sciences, for identification of specimens for this study.

\section{Authors' contributions}

SC and LX designed the study. YS. and DD performed most of the experiments and analyzed the data. YS and MY wrote the manuscript. LW, GD and DZ pro-

vided technical assistance. All authors read and approved the final manuscript.

\section{Funding}

This work was supported by Nonprofit industry research subject (No. 201507002-4-1-3)

\section{Availability of data and materials}

The materials are available from the corresponding author on reasonable reguest.

\section{Ethics approval and consent to participate}

All experiments were performed in accordance with the guidelines for the use and care of animals of the Center for Laboratory Animal Care, China Academy of Chinese Medical Sciences. All experimental protocols were approved by the Research Ethics Committee of Institute of Chinese Materia Medica, China Academy of Chinese Medical Sciences, Beijing, China.

\section{Consent for publication}

Not applicable.

\section{Competing interests}

The authors declare that they have no conflict of interest.

\section{Author details}

${ }^{1}$ Institute of Chinese Materia Medica, China Academy of Chinese Medical Sciences, Beijing 100700, China. ${ }^{2}$ Amway (China) Botanical Research and Development Center, Wuxi 214145, China. ${ }^{3}$ College of Agricultural and Environmental Sciences, University of California, Davis, CA 95616, USA.

Received: 22 June 2020 Accepted: 15 September 2020

Published online: 29 September 2020

References

1. Yuan J, et al. The textual research of Chinese material medica Gekko gecko. Chin Med Mat. 2011:34(3):474-7.

2. Chinese Pharmacopoeia Commission. Pharmacopoeia of the People's Republic of China. 1st ed. Beijing: China Medical Science Press; 2015. p. $343-4$

3. Zang H, et al. Chemical constituents and pharmacological actions of Gekko gecko Linnaeus. Jilin J Tradit Chin Med. 2016;36(9):919-21.

4. Liao C, et al. Effect of black-spotted geckos on immune regulation in mouse models of asthma. Chin Tradit Pat Med. 2014;36(10):2037-40.

5. You Q, et al. Anti-tumor effect and influence of Gekko gecko Linnaeus on the immune system of sarcoma 180-bearing mice. Mol Med Rep. 2009;2(4):573-7.

6. Wang G. Effect of ginseng gecko powder on the pulmonary function and quality of life in patients with stable COPD. Acta Chin Med. 2017:32(2):198-200.

7. Zou J, et al. Study on pharmacodynamics and toxicology of gejie dingchuan capsule. Chin Tradit Herbal Drugs. 2003;34(4):343-6.

8. Li H, et al. Resources and protect of Gekko gecko in Guangxi. J. Guangxi Normal Univ. 1996;2(14):64-8.

9. Caillabet O. The Trade in Tokay Geckos Gekko gecko in South-East Asia with a case study on novel medicinal claims in peninsular malaysia. Traffic. 2013; 2-8.

10. Zhu H, et al. Pharmacognostic identification of Gecko (Gekko gecko) and its counterfeits on characteristics of crude drug and original animal of 18 samples. Guangxi J Tradit Chin Med. 1999;22(1):39-43.

11. Zhang $\mathrm{H}$, et al. Identification of Gekko gecko Linnaeus and adulterants using the COI barcode. Mod Tradit Chin Med Mater Med World Sci Tech. 2014;2(16):269-73.

12. Wagner P, et al. Medicinal use of Gekko gecko (Squamata: Gekkonidae) has an impact on agamid lizards. Salamandra. 2014:50(3):185-6.

13. Bauer A. Geckos in traditional medicine: forensic implications. Appl Herpetol. 2009;6(1):81-96. 
14. Szydlowski P, et al. Histology and ultrastructure of the integumental chromatophores in tokay gecko (Gekko gecko) (linnaeus, 1758) skin. Zoomorphology. 2017;136(2):233-40.

15. Daza J, et al. Peramorphic traits in the tokay gecko skull. J Morphol. 2015;276(8):915-28.

16. Shen $L$, et al. Identification of Gekko gecko Linnaeus. Tianjin J Tradit Chin Med. 2010;27(6):518-9.

17. Chen $\mathrm{S}$, et al. A renaissance in herbal medicine identification: from morphology to DNA. Biotechnol Adv. 2014;32(7):1237-44.

18. Jia J, et al. Identification of antler powder components based on DNA barcoding technology. Acta Pharm Sin. 2015;50(10):1356-61.

19. Xiang $L$, et al. The species traceability of the ultrafine powder and the cell wall-broken powder of herbal medicine based on DNA barcoding. Acta Pharm Sin. 2015;50(12):1660-7.

20. Zhi Z, et al. Powder X-ray diffraction analysis of Chinese medicinal materials Gekko gecko. Chin Tradit Herbal Drugs. 2001;32(10):932-3.

21. Xu Y, et al. Prime study of Gekko gecko in HPLC fingerprint. Chin Med Mat. 2012;35(11):1735-8

22. Gu H, et al. Authentication of Chinese crude drug gecko by DNA barcoding. Nat Prod Commun. 2011;6(1):67-71.

23. Lamendin $R$, et al. Labelling accuracy in tasmanian seafood: an investigation using DNA barcoding. Food Control. 2015;47:436-43.

24. Meusnier I, et al. A universal DNA mini-barcode for biodiversity analysis. BMC Genomics. 2008;9(1):214

25. Doña J, et al. DNA barcoding and minibarcoding as a powerful tool for feather mite studies. Mol Ecol Resour. 2015;15(5):1216-25.

26. Shokralla S, et al. Pyrosequencing for mini-barcoding of fresh and old museum specimens. PLoS ONE. 2011;6(7):e21252.

27. Hellberg $R$, et al. Identification of meat and poultry species in food products using DNA barcoding. Food Control. 2017;80:23-8.

28. Chen SL, Standard DNA. Barcodes of Chinese Materia Medica in Chinese Pharmacopoeia. Beijing: Science Press; 2015. p. 19-20.

29. Folmer O, et al. DNA primers for amplification of mitochondrial cytochrome c oxidase subunit I from diverse metazoan invertebrates. Mol Mar Biol Biot. 1994;3(5):294-9.

30. SuY, et al. Identification of common edible and medicinal mushrooms by DNA barcoding. World J Tradit Chin Med. 2016;2(3):10-6.

31. Boyer $\mathrm{S}$, et al. Sliding window analyses for optimal selection of minibarcodes, and application to 454-pyrosequencing for specimen identification from degraded DNA. PLoS ONE. 2012;7(5):e38215.

32. Shokralla S, et al. A DNA mini-barcoding system for authentication of processed fish products. Sci Rep. 2015:5:15894.

33. Wang $X$, et al. A nucleotide signature for the identification of Angelicae sinensis radix (danggui) and its products. Sci Rep. 2016;6:34940.

34. Janjua $\mathrm{S}$, et al. DNA mini-barcoding for wildlife trade control: a case study on identification of highly processed animal materials. Mitochondrial DNA A. 2016:27(3):1-3.

35. Song $M$, et al. Identification of processed Chinese medicinal materials using DNA mini-barcoding. Chin J Nat Med. 2017;15(7):481-6.

36. Liu Z, et al. Authentication of Chinese crude drug, gecko, by allele-specific diagnostic PCR. Planta Med. 2001;67(4):385-7.

37. Gu H, et al. Authentication of gecko by allele-specific PCR. Sichuan J Zool. 2012;31(2):226-31.

38. Jiang $C$, et al. Molecular authentication of gecko and its adulterants by rapid PCR. Mod Chin Med. 2017;19(1):21-5

39. Amaral J, et al. Identification of duck, partridge, pheasant, quail, chicken and turkey meats by species-specific PCR assays to assess the authenticity of traditional game meat Alheira sausages. Food Control. 2015:47:190-5.

40. Fields $A$, et al. A novel mini-DNA barcoding assay to identify processed fins from internationally protected shark species. PLOS ONE. 2015:10(2):e0114844.
41. Amaral J, et al. Authentication of a traditional game meat sausage (Alheira) by species-specific PCR assays to detect hare, rabbit, red deer, pork and cow meats. Food Res Int. 2014;60(6):140-5.

42. Barakat $\mathrm{H}$, et al. Detection of pork adulteration in processed meat by species-specific PCR-QIAxcel procedure based on D-loop and cytb, genes. Appl Microbiol Biot. 2014;98(23):9805-16.

43. Pinto $A$, et al. Occurrence of mislabeling in meat products using DNAbased assay. J Food Sci Tech. 2015;52(4):2479-84.

44. Yuan J, et al. The textual research of black-spotted tokay gecko and redspotted tokay gecko. Chin Med Mat. 2008;31(9):1437-9.

45. Yu X, et al. Geographic variation in the advertisement calls of Gekko gecko in relation to variations in morphological features: implications for regional population differentiation. Ethol Ecol Evol. 2011;23(3):211-28.

46. Zhang Y, et al. A molecular phylogeny of red tokay and black tokay (Gekko gecko) based on mitochondrial $12 \mathrm{~S}$ rRNA gene sequences. Guangxi Med J. 2006;28(6):793-6.

47. Qin X, et al. Complete mitochondrial genome of the red-spotted tokay gecko (Gekko gecko, reptilia: gekkonidae): Comparison of red- and blackspotted tokay geckos. Mitochondr DNA. 2011;22(5-6):176-7.

48. Qin X, et al. Genetic variation and differentiation of Gekko gecko from different populations based on mitochondrial cytochrome b gene sequences and karyotypes. Zool Sci. 2012;29(6):384-9.

49. Wang $G$, et al. Nuclear and mitochondrial DNA reveals significant intraspecific genetic differentiation of tokay gecko in southern China and northern Vietnam. J Zool. 2012;287(3):215-23.

50. Gao S, et al. Isolation and characterization of novel microsatellite markers in Gekko gecko (Reptilia: Gekkonidae). Conserv Genet Resour. 2011;3(3):467-9.

51. Qin X, et al. RAPD analysis on genetic divergence and phylogenesis of Gekko gecko from different areas. Chin J Zool. 2005;40(6):14-8.

52. Kongbuntad W, et al. Genetic diversity of the red-spotted tokay gecko (Gekko gecko linnaeus, 1758) (squamata: Gekkonidae) in Southeast Asia determined with multilocus enzyme electrophoresis. J Asia-Pac Biodivers. 2016;9:63-8.

53. Zhang Y, et al. Insights from ecological niche modeling on the taxonomic distinction and niche differentiation between the black-spotted and redspotted tokay geckoes (Gekko gecko). Ecol Evol. 2014;4(17):3383-94.

54. Chinese Pharmacopoeia Commission. Pharmacopoeia of the People's Republic of China. 1st ed. Beijing: China Medical Science Press; 2010. p. 323.

55. Kim M, et al. Species identification of commercial jerky products in food and feed using direct pentaplex PCR assay. Food Control. 2017:78:1-6.

56. Sun W, et al. The potential power of Bar-HRM technology in herbal medicine identification. Front Plant Sci. 2016;7(e8613):367.

57. Jin Y, et al. High-resolution melting (HRM) analysis: a highly sensitive alternative for the identification of commercially important Crassostrea oysters. J Mollus Stud. 2014:81(1):167-70.

58. Ganopoulos I, et al. Universal ITS2 barcoding DNA region coupled with high-resolution melting (HRM) analysis for seed authentication and adulteration testing in leguminous forage and pasture species. Plant Mol Biol Rep. 2012;30(6):1322-8.

59. Buddhachat $K$, et al. Authenticity analyses of Phyllanthus amarus using barcoding coupled with HRM analysis to control its quality for medicinal plant product. Gene. 2015;573(1):84-90.

\section{Publisher's Note}

Springer Nature remains neutral with regard to jurisdictional claims in published maps and institutional affiliations. 